\title{
Analysis of Tumor Vascularization with Smooth Muscle Actin by Immunohistochemistry_it's Prognostic Significance in Differentiating Benign and Malignant Ovarian Surface Epithelial Tumors
}

\author{
Dr. Shyamala Srujana ${ }^{1 *}$, Dr. SSS Quadri ${ }^{1}$, Dr. N.Srimani ${ }^{2}$
}

\author{
${ }^{1}$ Associate Professor Department of Pathology, Government Medical College, Mahabubnagar Telangana India \\ ${ }^{2}$ Professor Department of Pathology, Gandhi Medical College, Secunderabad Telangana India
}

\author{
DOI: $10.36348 /$ SJPM.2019.v04i10.014 \\ | Received: 28.08.2019 | Accepted: 05.09.2019| Published: 30.10.2019 \\ *Corresponding author: Dr. Shyamala Srujana
}

\section{Abstract}

Ovarian carcinomas represent sixth most common female cancers and fourth leading cause of death due to cancers in women. Surface epithelial tumors are commonest variety of ovarian tumors followed by germ cell tumors. Angiogenesis is critical factor in tumor growth and metastasis, because tumor proliferation is severely limited by nutrient supply to proliferating tumor cells. Hence tumorigenesis of malignant neoplasms is associated with extensive neovascularization. Many studies has established micro vessel density as an important prognostic factor in solid tumors, but there is paucity of literature regarding characteristics of tumor blood vessels in ovarian surface epithelial tumors Aims and objectives: To clarify the differences in angiogenesis between benign and malignant epithelial tumors. To assess the characteristics of tumor vessels with aid of immunohistochemistry. Material and methods: The study was done on 30 specimens received at Gandhi hospital during May 2017-July 2018. All oophorectomy specimens received at department of pathology after considering the exclusion criteria were included in the study. Routine investigations, ultrasound abdomen and pelvis, $\mathrm{H}$ and E staining, immunohistochemistry Smooth Muscle Actin was performed. Smooth Muscle Actin expression is classified as high, moderate, low with normal ovary as control. Results: Out of 30 cases, 15 are benign (50\%), 3 borderline (10\%) and 12 cases are malignant (40\%). Youngest age at diagnosis was 29 years and oldest was at 65 years. Majority of the cases were diagnosed as serous tumors. Conclusion: Smooth muscle actin is highly expressed in benign cases, moderate in borderline and low in malignant cases. Malignant ovarian tumours statistically showed higher production of immature blood vessels along with paucity of smooth muscle support as compared to benign tumours. Therefore Mean MVD is higher and Smooth muscle actin expression is low in malignant tumors.

Keywords: Angiogenesis, Epithelial tumours, Micro Vessel density, ovary, smooth muscle actin

\begin{abstract}
Copyright @ 2019: This is an open-access article distributed under the terms of the Creative Commons Attribution license which permits unrestricted use, distribution, and reproduction in any medium for non-commercial use (NonCommercial, or CC-BY-NC) provided the original author and sources are credited.
\end{abstract}

\section{INTRODUCTION}

Ovarian carcinomas accounts for $3 \%$ of all cancers in females [1]. Ovarian carcinomas represent sixth most common female cancers and fourth leading cause of death due to cancers in women. Surface epithelial tumours are commonest variety of ovarian tumours followed by germ cell tumours [2]. Ovarian neoplasms have become increasingly important not only because of the large variety of neoplastic entities but more so because they have gradually increased mortality due to female genital cancers. Surface epithelial tumors were commonest variety of ovarian tumors followed by germ cell tumors. The common histological types of surface epithelial tumours are serous and mucinous tumours[3]. The surface epithelium consists of single layer of modified mesothelial cells varying from flat to cuboidal to columnar. Stroma consists of spindle shaped cells with scant cytoplasm arranged in storiform pattern. The peripheral zone of stroma is called as cortex which contains numerous follicles in various stages of development. The epithelial tumours are classified as benign, borderline, and malignant. The benign tumours are often subclassified based on components of tumours, which include cystic areas (cystadenomas), cystic and fibrous areas (cystadenofibromas), and predominantly fibrous areas (adenofibromas). The borderline and malignant tumours can have cystic component [1].

Angiogenesis is a critical factor in tumor growth and metastasis, because tumor proliferation is severely limited by nutrient supply to proliferating tumor cells. Hence tumorigenesis of malignant 
neoplasms associated with extensive neovascularization. The analysis of tumor vascularization by microvessel density (MVD) and its prognostic significance has been evaluated in many tumors including ovary. Smooth muscle actin stains a coat of periendothelial support cells called as pericytes and smooth muscle cells [15]. In normal ovaries, alpha smooth muscle actin is found in blood vessel walls, muscle fibers and stromal cells surrounding follicles [4]. It is possible to distinguish between newly formed immature vessels in malignant tumours and mature established vessels in benign tumours by use of antibodies to smooth muscle actin [5].

\section{AIMS AND OBJECTIVES}

- To determine the incidence of ovarian surface epithelial tumours at our hospital.

- To study the differences in angiogenesis between benign and malignant epithelial tumours using immunohistochemistry

- To assess characteristics of tumour vessels with aid of immunohistochemistry.

\section{MATERIALS AND METHODS}

Cross sectional study for a period of 15 months (From May 2017 to July 2018) Conducted at Department of Pathology, Gandhi Hospital/Gandhi Medical College, Secunderabad. Specimens of ovarian tumors received from Departments of Surgery and OBG Gandhi Hospital/Gandhi Medical College, Secunderabad and Departments of OBG, Government maternity Hospital, Petlaburg, Hyderabad were included in the study. After detailed gross examination of the specimens sections were given for histopathological examination. Immunohistochemistry is performed using standard protocol methods.
Reagents:- Antigen retrieval solution: citrate buffer, $\mathrm{pH}$ 6.0, Sodium chloride, 3\% Hydrogen peroxide, Diaminobenzidine (DAB) reagent, Harris hematoxylin and Primary antibody-smooth muscle actin (SMA)antibody (Dako). Smooth muscle actin expression in tumour vessels is classified as high, moderate, low, absent in comparison to normal ovary as control.

In each section, three most vascular areas were chosen using low power field (10x) - Hot spots. In each hot spot, SMA expression index is calculated and average of the three is done. SMA expression index is the ratio of tumour vessels with high or moderate intensity of SMA to number of all countable vessels at 40x [6].

\section{OBSERVATIONS AND RESULTS}

Incidence of ovarian surface epithelial tumours at our hospital is $48.37 \%$. Serous tumours are most common tumours $(60 \%)$ followed by mucinous tumours $(40 \%)$. Youngest age at diagnosis -29 years; oldest -65 years. Most of the patients belong to age group -40 to 49 years -20 cases.

\section{Among 30 cases of Ovarian Surface epithelial tumours}

- $\quad$ Benign cases - $15(50 \%)$,

- Borderline cases - $3(10 \%)$ and

- Malignant cases - $12(40 \%)$.

Among the tumours,

- $\quad$ Serous tumors- 18 cases $(60 \%)$

- Mucinous tumours -11 cases ( $37 \%$ ) and

- Brenner tumours-1 cases $(3 \%)$

Table-1: Histomorphological Distribution of Surface Epithelial Tumours of Ovary

\begin{tabular}{|l|c|c|c|}
\hline \multicolumn{1}{|c|}{ Type of Tumour } & $\begin{array}{c}\text { Benign } \\
\mathbf{( 5 0 \% )}\end{array}$ & $\begin{array}{c}\text { Borderline } \\
(\mathbf{1 0 \%})\end{array}$ & $\begin{array}{c}\text { Malignant } \\
\mathbf{( 4 0 \% )}\end{array}$ \\
\hline $\begin{array}{l}\text { SEROUS TUMOURS } \\
\mathrm{n}=18(60 \%)\end{array}$ & 10 & 02 & 06 \\
\hline $\begin{array}{l}\text { MUCINOUS TUMOURS } \\
\mathrm{n}=11(37 \%)\end{array}$ & 07 & 01 & 03 \\
\hline $\begin{array}{l}\text { BRENNER TUMOURS } \\
\mathrm{N}=1(3 \%)\end{array}$ & 00 & -- & 01 \\
\hline
\end{tabular}

Table-2: Age Distribution

\begin{tabular}{|c|c|}
\hline Age & Number of Patients \\
\hline $20-29$ & 01 \\
\hline $30-39$ & 03 \\
\hline $40-49$ & 20 \\
\hline $50-59$ & 05 \\
\hline $60-69$ & 01 \\
\hline Total & 30 Cases \\
\hline
\end{tabular}

Smooth muscle actin is used commonly as a marker of myofibroblast formation. Alpha smooth muscle actin stains pericytes and smooth muscle cells in blood vessels. Alpha-smooth muscle actin is present in vascular smooth muscle cells in high amounts. It is demonstrated in pericytes of cytoplasm. In smooth muscle cells and pericytes, alpha smooth muscle is localized in microfilaments.

Blood vessels formation is important in pathogenesis of rapid growth and metastasis of solid tumours. Several angiogenic factors have been identified like basic and acidic fibroblast growth factor, angiogenin, VEGF, elevated serum VEGF values in ovarian cancer patients are correlating with tumour 
differentiation, metastasis and more significantly shorter survival time [5]. If a solid tumour has all the genetic aberrations required for malignant transformation, it cannot enlarge beyond 1-2 $\mathrm{mm}$ in diameter unless it induces angiogenesis. Tumours require oxygen and nutrients and removal of waste products and maximum distance from blood vessels from which they can diffuse is $1-2 \mathrm{~mm}$. So growing tumours stimulate neoangiogenesis, vessels sprout from pre-existing capillaries [7]. In absence of vascular support, tumours may become apoptotic or necrotic [8]. Neoangiogenesis has double effect on tumour growth: (1). It supplies needed nutrients and oxygen (2). Newly formed endothelial cells stimulate growth of adjacent tumour cells by secreting insulin like growth factors and PDGF [9].

These vessels are not normal and are dilated; leaky having haphazard patterned of connection with fragmented basement membrane Neo-angiogenesis contributes to metastasis by permitting access to tumour cells. Thus, angiogenesis is an essential facet of malignancy [10].

- Smooth muscle actin expression in tumour vessels is classified as high, moderate, low, absent in comparison to normal ovary as control.

- In each section, three most vascular areas were chosen using low power field (10x) - Hot spots.

- In each hot spot, SMA expression index is calculated and average of the three is done.

- SMA expression index is the ratio of tumour vessels with high or moderate intensity of SMA to number of all countable vessels at 40x [6].

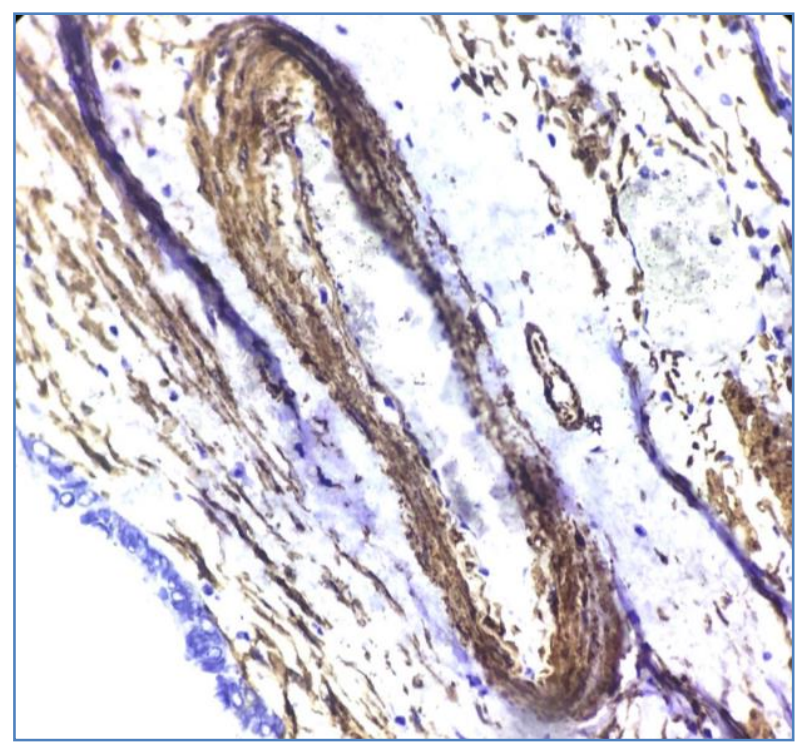

Fig-1: IHC Stain-SMA- 40X- Benign Serous Cystadenoma showing continuous, complete intense, staining of blood vessel wall

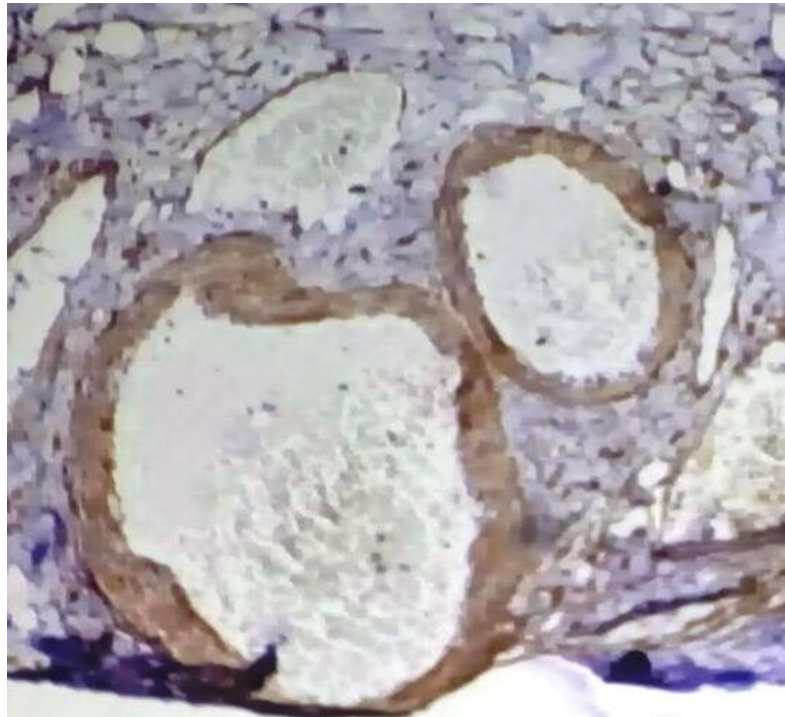

Fig-2: IHC Stain- SMA - 40X- Benign mucinous cystadenoma showing continuous, complete intense, staining of blood vessel wall

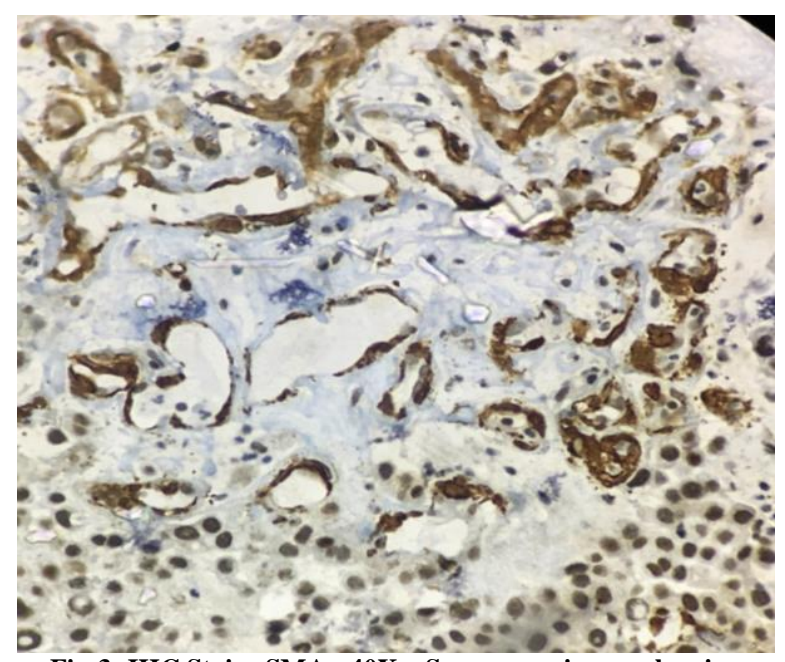

Fig-3: IHC Stain- SMA - 40X - Serous carcinoma showing discontinuous, incomplete, weak staining of blood vessel wall

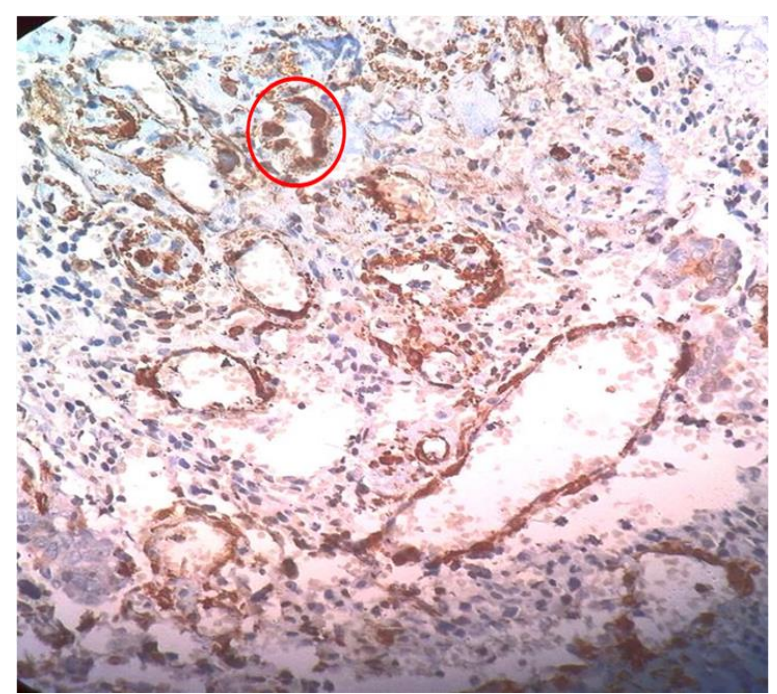

Fig-4: IHC Stain - Smooth muscle actin- 40X serous carcinoma showing moderate intensity vessel which is highlighted 


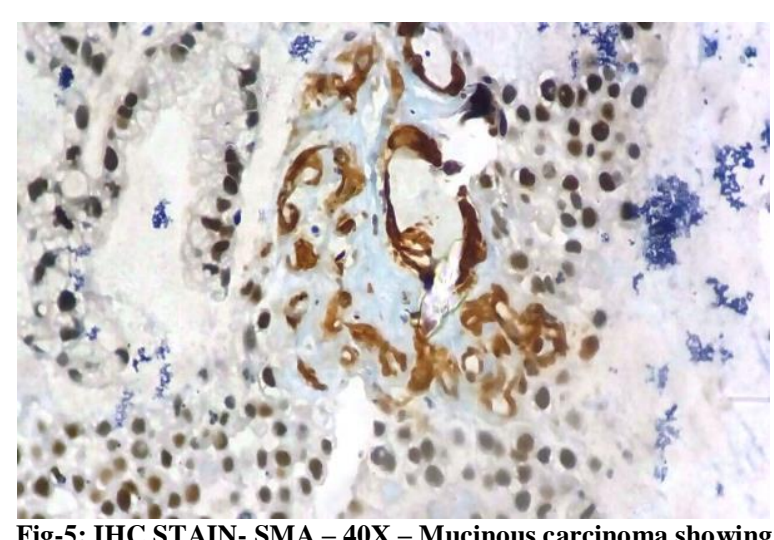

Fig-5: IHC STAIN-SMA - 40X - Mucinous carcinoma showing discontinuous, incomplete, weak staining of blood vessel wall

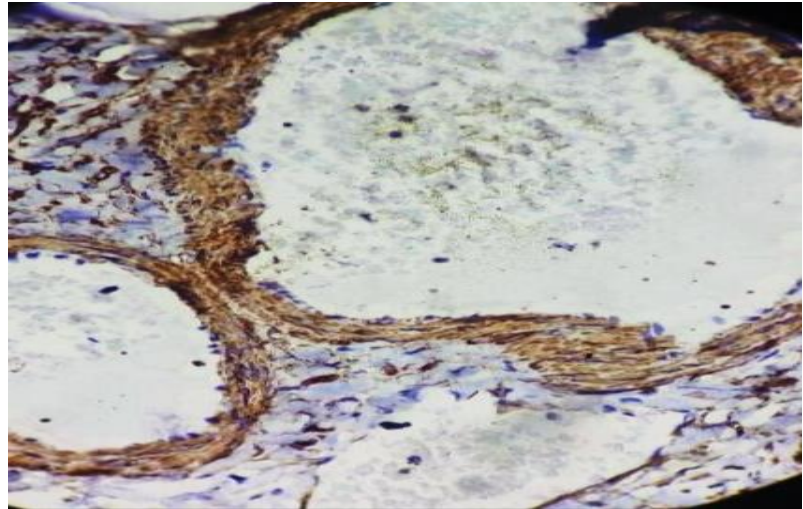

Fig-6: IHC Stain- SMA- 40X- Benign mucinous cystadenoma showing continuous, complete intense, staining of blood vessel wall

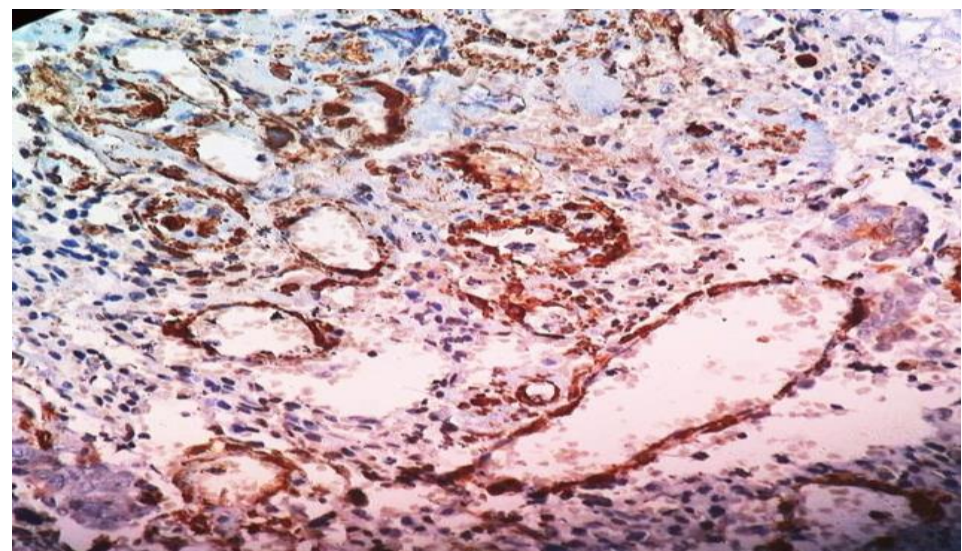

Fig-7: IHC Stain - SMA - 40X - Mucinous carcinoma showing discontinuous, incomplete, weak staining of blood vessel wall

Table-3: Smooth Muscle Actin Expression index of Benign Ovarian Surface Epithelial Tumours

\begin{tabular}{|c|c|c|}
\hline Benign Tumours & Number of Cases & Mean SMA Expression Index(SD) \\
\hline Serous Cystadenoma & 10 & 0.46 \\
\hline Mucinous Cystadenoma & 05 & 0.496 \\
\hline Total & 15 & $0.48(0.08)$ \\
\hline
\end{tabular}

NOTE: Mean Smooth Muscle Actin Expression index of Benign Ovarian Surface Epithelial tumours is $0.48 \pm 0.08$

Table-4: Smooth Muscle Actin Expression Index of Borderline Ovarian Surface Epithelial Tumours

\begin{tabular}{|c|c|c|}
\hline Borderline Tumours & Number of Cases & Mean SMA Expression Index \\
\hline Serous Borderline Tumour & 02 & 0.286 \\
\hline Mucinous Borderline Tumour & 01 & 0.32 \\
\hline Total & 03 & 0.303 \\
\hline
\end{tabular}

NOTE: Mean Smooth Muscle Actin expression index of Borderline Ovarian Surface Epithelial Tumours is 0.303 calculated

As only 3 cases of borderline ovarian surface epithelial tumours are included, standard deviation is not

Table- 5: Smooth Muscle Actin Expression Index of Malignant Ovarian Surface Epithelial Tumours

\begin{tabular}{|c|c|c|}
\hline Malignant Tumours & Number of Cases & Mean SMA Expression Index(SD) \\
\hline Serous Carcinoma & 06 & 0.152 \\
\hline Mucinous Carcinoma & 05 & 0.128 \\
\hline Malignant Brenner Tumour & 01 & 0.214 \\
\hline Total & 12 & $0.17(0.03)$ \\
\hline
\end{tabular}

NOTE: Mean Smooth Muscle Actin Expression Index of Malignant Ovarian Surface Epithelial Tumours is $0.17 \pm 0.03$ 
Table-6: Comparision of Smooth Muscle Actin expression index of Ovarian Surface Epithelial Tumours

\begin{tabular}{|c|c|}
\hline Type of Tumour & Mean SMA \\
\hline Benign & O.48 \\
\hline Borderline & 0.303 \\
\hline Malignant & 0.17 \\
\hline & \\
\hline
\end{tabular}

Mean Smooth Muscle Actin Expression Index of malignant ovarian surface epithelial tumours (0.17) is low as compared to benign and borderline tumours

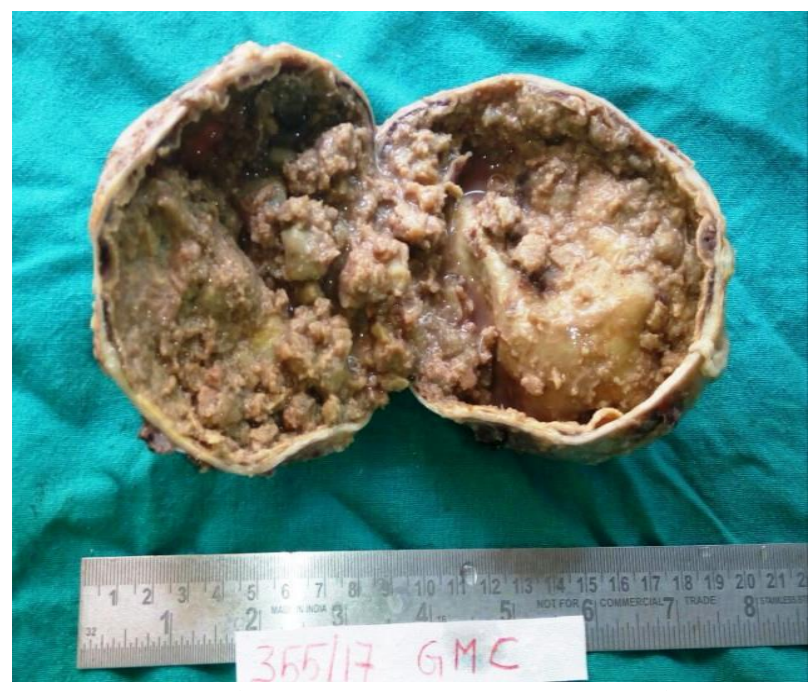

Fig-8: Gross Specimen - cut surface of Papillary Serous Carcinoma showing papillary fronds

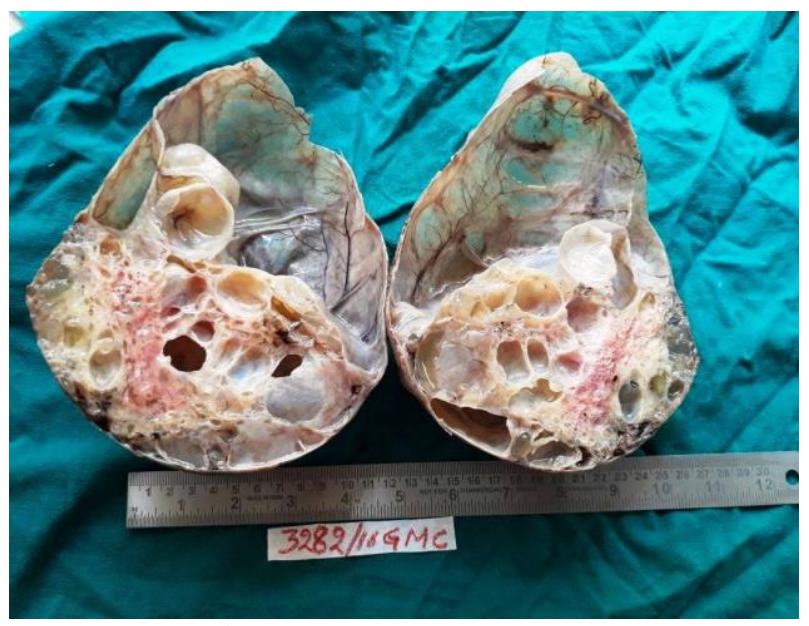

Fig-9: Gross Specimen - Cut surface of Benign Mucinous Cystadenoma showing multilocular cyst wall with mucinous areas

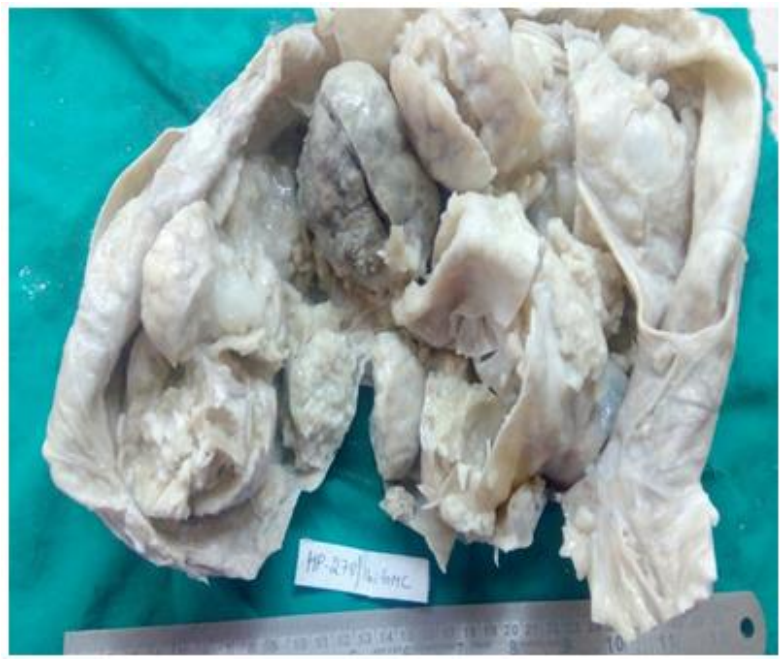

Fig-10: Gross Specimen-Cut surface of malignant Brenner tumour showing grey white solid areas

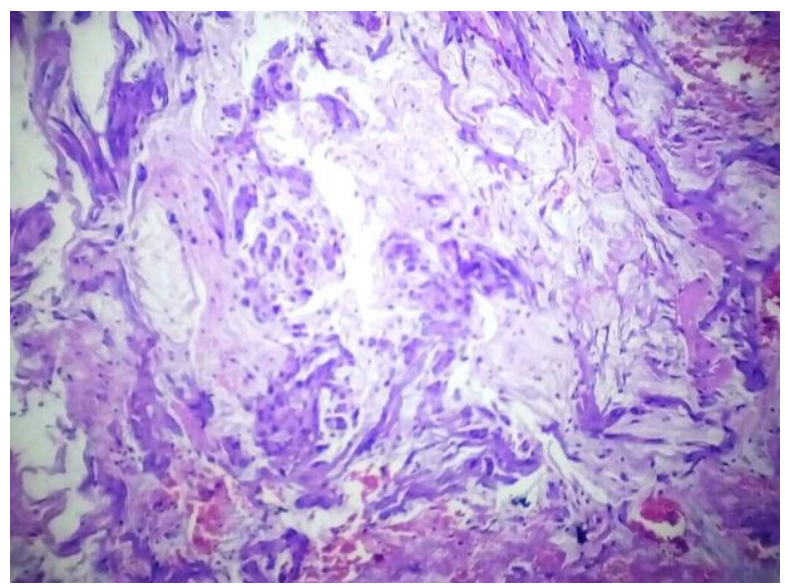

Fig-11: H \& E stain- 40X - Mucinous Carcinoma showing pools of mucin

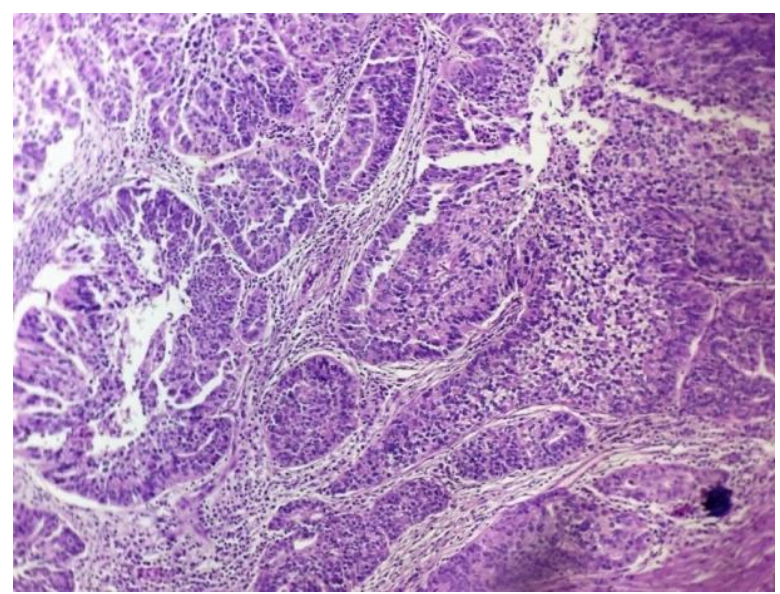

Fig-12: H\&E Stain-40X- malignant Brenner tumour showing malignant transitional epithelium with focus of benign island

\section{DISCUSSION}

Ovarian carcinomas represent sixth most common female cancers and fourth leading cause of death due to cancers in women. Surface epithelial tumours are commonest variety of ovarian tumours followed by germ cell tumours[2]. Ovarian neoplasms have become increasingly important not only because 
of the large variety of neoplastic entities but more so because they have gradually increased mortality due to female genital cancers. Surface epithelial tumors were commonest variety of ovarian tumors followed by germ cell tumors. The aim of the present study is to evaluate the microvessel density and maturity characteristics of proliferating blood vessels in benign and malignant surface epithelial tumours of ovary. The results thus obtained are analyzed and discussed in the light of other publications. Microvessel density is significantly higher in malignant ovarian surface epithelial tumours compared to benign tumours. Blood vessels of malignant ovarian surface epithelial tumours have decreased Smooth Muscle Actin expression as compared to benign tumours. Malignant tumours showed immature blood vessels and paucity of smooth muscle support. These results reveal that blood vessels in malignant tumours are thin walled, weak and haphazardly arranged, allowing easier spread of tumour cells.

In regard to Smooth muscle actin expression index, it is higher than malignant tumours and lower than benign tumours. As the sample size is too low, it is difficult to know any statistical significance. More sample size is required and further studies are recommended for evaluation. Hypoxia triggers angiogenesis by HIF-1 $\alpha$ on transcription of proagiogenic factors VEGF. Tumours initially do not require an extensive vasculature obtaining nutrients through diffusion. This prevascular phase can maintain the tumour only to a certain size. For the tumour to attain the size greater than $2 \mathrm{~mm}$ diameter neovascularization must occur $[11,12]$.

Table-7: Supporting Studies of Smooth Muscle Actin expression index

\begin{tabular}{|c|c|c|c|}
\hline Study & SMA Expression Index - Benign & SMA Expression Index - Malignant & P Value \\
\hline Present study & 0.48 & 0.17 & $<0.001$ \\
\hline Emoto et al. [61] & 0.69 & 0.18 & $<0.001$ \\
\hline
\end{tabular}

Induction of angiogenesis is also important in the process of carcinogenesis. The acquisition of angiogenic capability can be seen in ovarian peritoneal implants, which remain small and avascular until neovascularization from adjacent peritoneal vessels occurs. Angiogenesis and neoplastic transformation are not interdependent and each one can occur in the absence of other [13-15]. The nature of proliferating blood vessels and its role in regulating the biological behavior of a neoplasm can be assessed to some extent by microvessel density and smooth muscle actin expression in blood vessels. The degree of angiogenesis of a tumour as assessed by micro vessel density has emerged as a powerful candidate for prognosis and as predictive tool. Studies done in breast cancer [8] and cutaneous melanomas [50] have established microvessel density as an important prognostic indicator. Few studies show link between micro vessel density and prognosis in solid tumours like nonsmall cell lung carcinoma, esophagus, prostate, ovary[16-18]. Micro vessel density is the mean value of micro vessel count derived using specific objective magnification in three or four fields, with known field diameter. It is subjectively selected from "hot spots". To distinguish between newly formed vessels from mature vessels especially in benign tumours, large vessels with thick muscular wall and dilated lumina were excluded from the count as proposed by Bosari et al. Regarding smooth muscle actin expression in ovarian surface epithelial tumours various studies have evaluated the significance of its expression in benign and malignant tumours. In our study, smooth muscle actin expression index of malignant surface epithelial tumours is less compared to benign and borderline tumours.
Emoto et al. [19] studied on 106 patients about characteristics of vessels by using antibodies against smooth muscle actin. Study shows that vessels of malignant tumours demonstrated poor SMA expression compared with vessels of benign tumours.

\section{CONCLUSION}

- Incidence of ovarian surface epithelial tumours at our hospital is $48.37 \%$. Serous tumours are most common tumours $(60 \%)$ followed by mucinous tumours. $(40 \%)$

- Malignant ovarian tumours showed higher production of immature blood vessels along with paucity of smooth muscle support as compared to benign tumours. Therefore Mean Micro Vessel Density is higher and Smooth muscle actin expression is low in malignant tumours as compare to benign tumours.

- Blood vessels in malignant tumours are thin walled, weak and haphazardly arranged, thus allowing easier spread of tumour cells.

- Long term followup studies are needed to see if Micro Vessel Density and Smooth Muscle Actin expression index are of prognostic value.

- In view of these results, new therapy should aim to alter ratio of immature to mature blood vessels and also Micro vessel density of malignant tumours for a successful outcome.

\section{REFERENCES}

1. Crum, C. P. (2004). The Female genital tract. Robbins and Cotran Pathologic Basis of Disease.

2. Kumar, R. V., Mukherjee, G., Umadevi, K., \& Hazarika, D. (1994). Sclerosing stromal tumour of the ovary. Indian journal of cancer, 31(2), 92-95. 
3. Krigman, H., Bentley, H., Robboy, S.J. (1994). Pathology of epithelian ovarian tumours. Clin Obstet Gynecol, 37:475-91.

4. Benjamin, L.E., Golijanin, D., Itin, A.(1999). Selective ablation of immatureblood vessels in established human tumours follows vascular endothelial growth factor withdrawal. J Clin Invest, 103:159-65.

5. Kobayashi, H., Tsuruchi, N., Sugihara, K., Kaku, T., Saito, T., Kamura, T., ... \& Taniguchi, S. I. (1993). Expression of $\alpha$-smooth muscle actin in benign or malignant ovarian tumors. Gynecologic oncology, 48(3), 308-313.

6. Sehgal, S., Goyal, P., Agarwal, R., Singh, S., Kumar, A., Gupta, R., ... \& Agrawal, D. (2013). Differences in the angiogenesis of benign and malignant ovarian surface epithelial tumors demonstrated by microvessel density and immunohistochemistry. $J \quad$ Interdiscipl Histopathol, 1(3), 145-152.

7. Kumar, V., Abbas, A. K., Fausto, N., \& Aster, J. (2005). Neoplasia. Robbins and Cotran pathologic basis of disease. Philadelphia, PA, 2014, 336.

8. Holmgren, L., O'Reilly, M. S., \& Folkman, J. (1995). Dormancy of micrometastases: balanced proliferation and apoptosis in the presence of angiogenesis suppression. Nature medicine, 1(2), 149.

9. Parangi, S., O'Reilly, M., Christofori, G., Holmgren, L., Grosfeld, J., Folkman, J., \& Hanahan, D. (1996). Antiangiogenic therapy of transgenic mice impairs de novo tumor growth. Proceedings of the National Academy of Sciences, 93(5), 2002-2007.

10. Nagy, J. A., Brown, L. F., Senger, D. R., \& Lanir, N. (1989). Van de WL, Dvorak AM, Dvorak HF: Pathogenesis of tumor stroma generation: a critical role for leaky blood vessels and fibrin deposition. Biochim Biophys Acta, 948, 305-326.
11. Paweletz, N., \& Knierim, M. (1989). Tumorrelated angiogenesis. Critical reviews in oncology/hematology, 9(3), 197-242.

12. Ribatti, D., Vacca, A., Roncali, L., \& Dammacco, F. (1991). Angiogenesis under normal and pathological conditions. Haematologica, 76(4), 311-320.

13. Folkman, J. (1992, April). The role of angiogenesis in tumor growth. In Seminars in cancer biology (Vol. 3, No. 2, pp. 65-71).

14. Folkman, J. (1971). Tumor angiogenesis: therapeutic implications. New england journal of medicine, 285(21), 1182-1186.

15. Folkman, J. (1990). Introduction. Cancer Metastasis Rev;9:171-4.

16. Srivastava, A., Laidler, P., Davies, R. P., Horgan, K., \& Hughes, L. E. (1988). The prognostic significance of tumor vascularity in intermediatethickness (0.76-4.0 mm thick) skin melanoma. A quantitative histologic study. The American journal of pathology, 133(2), 419.

17. Albo, D., Granick, M. S., Jhala, N., Atkinson, B., \& Solomon, M. P. (1994). The relationship of angiogenesis to biological activity in human squamous cell carcinomas of the head and neck. Annals of plastic surgery, 32(6), 588-594.

18. Ohta, Y., Endo, Y., Tanaka, M., Shimizu, J., Oda, M., Hayashi, Y., ... \& Sasaki, T. (1996). Significance of vascular endothelial growth factor messenger RNA expression in primary lung cancer. Clinical Cancer Research,2(8), 14111416.

19. Emoto, M., Iwasaki, H., Mimura, K., Kawarabayashi, T., \& Kikuchi, M. (1997). Differences in the angiogenesis of benign and malignant ovarian tumors, demonstrated by analyses of color Doppler ultrasound, immunohistochemistry, and microvessel density. Cancer: Interdisciplinary International Journal of the American Cancer Society, 80(5), 899-907. 